Preface

\title{
Editorial Compilation IV
}

\author{
Emmanuel J. Favaloro, PhD, FFSc (RCPA) ${ }^{1}$ Giuseppe Lippi, MD ${ }^{2}$ \\ ${ }^{1}$ Department of Haematology, Institute of Clinical Pathology and \\ Medical Research (ICPMR), Westmead Hospital, Westmead, New \\ South Wales, Australia \\ 2 Section of Clinical Biochemistry, University of Verona, Verona, Italy
}

Semin Thromb Hemost 2017;43:549-552.

Welcome to another issue of Seminars in Thrombosis $\mathcal{E}$ Hemostasis. This is the fourth issue published under the "banner" of "Editorial Compilation." Although Seminars in Thrombosis and Hemostasis is primarily a theme-driven publication, the opportunity intermittently arises to publish issues containing more wide-ranging articles of current interest and controversy, which do not quite match a current themed issue in progress. We also require a medium to enable publication of contributions from our Eberhard $\mathrm{F}$. Mammen Young Investigator Award winners. ${ }^{1-6}$ The current issue has a mixture of content that comprises all these elements, as well as broadly fitting within the separate themes of "thrombosis" and "bleeding."

This issue begins with the contributions from four of our most recent (2015 and 2016) Eberhard F. Mammen Young Investigator Award winners. ${ }^{5,6}$ The first, representing the contribution from Jiménez-Alcázar et $\mathrm{al}^{7}{ }^{7}$ is on the topic of circulating extracellular DNA (ceDNA), and poses the question of whether this is the cause or consequence of thrombosis? As is well known to our readers, thrombosis leads to ischemic organ damage, and potentially arises in many disease processes. Neutrophils promote thrombosis in vitro and in vivo by releasing neutrophil extracellular traps (NETs). NETs are composed of DNA filaments coated with histones and neutrophil enzymes such as myeloperoxidase (MPO). ceDNA is not only used in cancer diagnostics but also as a surrogate marker to monitor NET formation in thrombosis. This narrative review therefore summarizes the association of ceDNA with human thrombosis. Levels of ceDNA indicate the extent and outcome of several cardiovascular and thromboembolic diseases, including myocardial infarction, stroke, and venous thromboembolism. ceDNA correlates with markers of coagulation and platelet consumption, thus supporting the hypothesis that ceDNA may be a surrogate marker of thrombus formation. In addition, ceDNA levels correlate with markers of cell injury and size of ischemic lesions, thus suggesting that ceDNA does not derive from NETs but is probably released from damaged tissues. Thus, it remains to be established whether ceDNA derives from NETs or is a cause or consequence of thrombosis.

Continuing the theme of "thrombosis," another of the Young Investigators, Schreiber et $\mathrm{al}^{8}$ then describe the potential utility of a drug called hydroxychloroquine, to improve pregnancy outcome in women with antiphospholipid antibodies (aPL). Women with aPL are at risk of many adverse pregnancy outcomes, including recurrent first trimester pregnancy loss and late pregnancy complications such as preeclampsia, HELLP (hemolysis, elevated liver enzymes, low platelet count) syndrome, premature delivery, intrauterine growth restriction, placental abruption, and intrauterine death. Current standard of care in obstetric antiphospholipid syndrome includes aspirin and heparin, and has resulted in live-birth rates of $\sim 70 \%$. However, $30 \%$ of such patients continue to have pregnancy complications. Hydroxychloroquine is suggested as a new treatment approach, but no randomized controlled trials have assessed its efficacy in clinical practice. The authors summarize information on the use of hydroxychloroquine in this setting, as well as describing their plans for a randomized controlled trial to identify its true utility in pregnant women with persistent aPL.

The last two contributions from recent Young Investigators are in the area of bleeding disorders. First, Selvam and James discuss angiodysplasia in the setting of von Willebrand disease (VWD). ${ }^{9}$ Angiodysplasia can lead to severe and intractable gastrointestinal bleeding in up to $20 \%$ of patients with VWD. Currently, the lack of an optimal treatment for this recurrent problem presents an ongoing challenge for many physicians in their management of affected patients. Over the past few years, studies have pointed to a regulatory role for the hemostatic protein, von Willebrand factor (VWF), in angiogenesis, providing a novel target for the modulation of vessel development. The authors therefore review the
Address for correspondence Emmanuel J. Favaloro, PhD, FFSc (RCPA), Department of Haematology, Institute of Clinical Pathology and Medical Research (ICPMR), Westmead Hospital, Westmead, NSW, Australia (e-mail: emmanuel.

favaloro@health.nsw.gov.au).
Issue Theme Editorial

Compilation IV; Guest Editors:

Emmanuel J. Favaloro, PhD, FFSc (RCPA), and Giuseppe Lippi, MD.
Copyright $\odot 2017$ by Thieme Medical Publishers, Inc., 333 Seventh Avenue, New York, NY 10001, USA. Tel: +1(212) 584-4662.
DOI https://doi.org/ 10.1055/s-0037-1603985. ISSN 0094-6176. 
clinical implications and molecular pathology of angiodysplasia in VWD.

Next, Muczynski et al review the development of therapeutic strategies for the treatment of hemophilia $A .{ }^{10}$ Current replacement therapy, involving intravenous infusion of plasma derived or recombinant factor VIII (FVIII) has proven its efficiency to control bleeding episodes in affected patients. However, replacement therapy requires frequent infusions to maintain efficacy and represents a serious burden that impacts quality of life. Recent half-life extension in a first generation of long-acting FVIII variants with improved circulating survival may change the current status. Such molecules are in clinical phase III studies, or have already received approval for therapeutic use, and will now allow reductions in frequency of infusion and reduce the burden of treatment of hemophilia A. Nevertheless, halflife extensions of FVIII have not been as effective as expected, particularly in regard to results obtained with other therapeutic molecules, such as factor IX (FIX). To overcome these limitations, inherent to the nature of FVIII biology, alternate bypassing therapies independent of the FVIII molecule are currently in development. These emerging approaches exploit modulation of the coagulation/anticoagulation balance, to compensate for FVIII deficiency.

The next series of articles comprise either contributions from editorial members of Seminars in Thrombosis \& Hemostasis, or the best of recent unsolicited material. The first set of articles continues the initial "prothrombotic" theme. First, Stavropoulos et al pose the question-does hematocrit and stroke reflect a forgotten and neglected link? ${ }^{11}$ Stroke is considered among the most common causes of mortality and disability, leading to dramatic socioeconomic consequences. From a pathophysiologic perspective, enhanced blood viscosity due to increased hematocrit values might be associated with stroke through impaired cerebral blood perfusion. This association has remained rather neglected during previous decades, but has newly emerged as an epicenter of scientific interest due to the unexpected elevation of stroke rates with sodium-glucose cotransporter-2 inhibitors, a new class of hypoglycemic drugs with otherwise dramatic cardiovascular benefits. The purpose of this article is to review available data on the relationship between stroke and hematocrit. Data from large observational studies point toward an increased risk for stroke in individuals with elevated hematocrit values. Data also suggest that the coexistence of increased hematocrit values and hypertension significantly enhance the risk of cerebrovascular events compared with each condition alone. In addition, high hematocrit values seem related to worse survival outcomes in poststroke patients. Collectively, the association between hematocrit and stroke seems to be strong and independent in patients with high hematocrit values (i.e., $>0.50$ ) in both previously healthy individuals and poststroke patients, but remains less clarified in patients with normal hematocrit values.

Arachchillage and Laffan then address the question of whether the mean platelet volume (MPV) be used as a predictor of vascular disease? ${ }^{12}$ Platelets play a pivotal role in both hemostasis and thrombosis. Increased platelet vo- lume has several etiologies and may be secondary to a genetic variant, platelet activation, increased platelet turnover, or in response to inflammatory stimuli. There are several hereditary syndromes in which an increased MPV occurs in association with a reduction in platelet number and function. An acquired increase in platelet size is often associated with increased platelet reactivity, shortened bleeding time, and increased platelet aggregation. Many studies have shown that a high MPV value is associated with increased risk of venous and arterial thromboses, but genome-wide association analyses found a weak inverse association between the risk of coronary heart disease and MPV. The disease associations of MPV suggest it could provide a useful contribution to risk assessment algorithms for both venous thromboembolism and arterial thrombosis. However, due to the problems associated with reliably assessing MPV, the authors assert that it does not yet constitute a practically useful biomarker for this purpose, and that before MPV can be adopted for wider clinical use, preanalytical and methodological problems involved in obtaining accurate and interlaboratory comparable results must be resolved. In particular, a major degree of standardization is required, as are local reference ranges calculated with respect to specified time intervals from venipuncture to laboratory analysis and different instrumentation. The authors also discuss the potential role of MPV in predicting the risk of acquired thrombotic disorders in addition to roles in congenital thrombocytopenic disorders, as well as the problems and pitfalls associated with the use of MPV in these settings.

Lippi et al then explore the intriguing link between intestinal microbiota and cardiovascular disease. ${ }^{13}$ Human microbiota is a term conventionally used to define the normal flora of microbes living in all of us, most of which are resident in the gastrointestinal tract. Despite it having been known for some time that the vast majority of intestinal bacteria exert a strong influence on human life, recent technological breakthroughs have made it possible to more accurately characterize the host microbial communities and explore their relationship with many human diseases. Notably, the evidence accumulated over the past 10 years suggests that a reasonable relationship can now be established between gut microbiota composition and the risk of cardiovascular disease. The most convincing information comes from data generated by studies involving trimethylamine-N-oxide (TMAO)-producing bacteria. It seems now clear that these bacterial strains may actively contribute to increase the concentration of endogenous TMAO and consequently enhance the risk of ischemic and thrombotic disorders, therefore, opening intriguing scenarios for effective prevention of cardiovascular disease by targeting the intestine by means of diet, probiotics, prebiotics, antibiotics, or even transplantation of gut microbes.

Next, Dentali et al report a systematic review and metaanalysis of the literature around lipoprotein(a) (Lp(a)) as a risk factor for venous thromboembolism. ${ }^{14}$ Elevated plasma levels of this unique lipoprotein are associated with increased cardiovascular risk in several clinical studies. 
However, there is a lack of data supporting a positive association between elevated $\mathrm{Lp}(\mathrm{a})$ levels and venous thromboembolism. The authors therefore conducted a systematic review of the literature to better clarify the role of $\operatorname{Lp}(a)$ as a risk factor for venous thromboembolism. $\operatorname{Lp}(\mathrm{a})$ was slightly but significantly associated with an increased risk of venous thromboembolism and patients with venous thrombosis had significantly higher Lp(a) values compared with controls.

Lippi et al then report on an original study on growth differentiation factor-15 (GDF-15), ${ }^{15}$ a strong and independent predictor of cardiovascular events and mortality, albeit that pathophysiological mechanisms underlying the association remain speculative. This study aimed to investigate the potential associations between the serum concentration of GDF-15 and clinical or laboratory parameters in a population of ostensibly healthy subjects. The study population consisted of 44 healthy volunteers enrolled from the laboratory staff, who had their blood collected for assessing complete blood cell count, GDF-15, serum creatinine, albumin, cardiac troponin T, galectin-3, routine coagulation tests, D-dimer, VWF, and platelet function testing using Platelet Function Analyzer-100 (PFA-100). In univariate analysis, serum GDF15 was found to be positively associated with age and plasma fibrinogen, and negatively associated with renal function and collagen-epinephrine (CEPI). In multiple linear regression analysis, serum GDF-15 remained significantly associated with renal function, CEPI, and plasma fibrinogen. Healthy subjects with GDF-15 above the median value had a twofold probability of displaying shorter CEPI closure times. Taken together, these results suggest that higher serum values of GDF-15 may be associated with overall global platelet hyperactivity and increased plasma fibrinogen, therefore, providing another plausible explanation for the association among GDF-15, cardiovascular events, and mortality.

Finally, Liu et al report another original study, this time on the clinical significance of antinuclear and antiextractable nuclear antigen (ANA/A-ENA) antibodies in childhood immune thrombocytopenia (ITP). ${ }^{16}$ Overall, 1,330 patients aged between 1 and 16 years diagnosed with primary ITP were retrospectively analyzed, excluding those with secondary ITP. Bleeding manifestations were recorded. All patients underwent autoantibody testing and follow-up for 32 months on average (range, 23-54 months). Steroid response was also assessed. Response rates were compared between ANA/A-ENA-positive and ANA/A-ENA-negative patients. Patients who were ANA/AENA positive were more likely to be female and older than 10 years. Patients who were A-ENA positive were more likely to have either persistent or chronic disease, and suffer from lifethreatening bleeding as well as poor short-term therapeutic response. The authors conclude that autoantibody testing is important to determine the short-term prognosis of ITP patients, and recommend that females, patients older than 10 years of age, and patients with either mixed positivity or A-ENA positivity should be more closely monitored.
As always, we would like to thank all the authors to this latest issue of "Editorial Compilations" for their original and comprehensive contributions. We also hope that you, representing the readership of this journal, find this issue of substantial interest. This will, of course, be determined in time, as measured and established for previous issues of this journal.

\section{References}

1 Favaloro EJ. 2011 Eberhard F. Mammen award announcements. Semin Thromb Hemost 2011;37(05):431-439

2 Favaloro EJ. 2012 Eberhard F. Mammen award announcements. Semin Thromb Hemost 2012;38(05):425-432

3 Favaloro EJ. 2013 Eberhard F. Mammen award announcements. Semin Thromb Hemost 2013;39(06):567-574

4 Favaloro EJ. 2014 Eberhard F. Mammen award announcements: part II-Young Investigator Awards. Semin Thromb Hemost 2014; 40(07):718-723

5 Favaloro EJ. 2015 Eberhard F. Mammen award announcements: part II - Young Investigator Awards. Semin Thromb Hemost 2015; 41(08):809-815

6 Favaloro EJ. 2016 Eberhard F. Mammen Award Announcements: part II-Young Investigator Awards. Semin Thromb Hemost 2017; 43(03):235-241

7 Jiménez-Alcázar M, Kim N, Fuchs TA. Circulating extracellular DNA - cause or consequence of thrombosis? Semin Thromb Hemost 2017;43(06):553-561

8 Schreiber K, Breen K, Cohen $\mathrm{H}$, et al. HYdroxychloroquine to improve Pregnancy outcome in women with AnTIphospholipid Antibodies (HYPATIA) protocol: a prospective phase IV multicentre randomised controlled trial of hydroxychloroquine vs placebo in addition to standard treatment in pregnant women with antiphospholipid syndrome or antibodies. Semin Thromb Hemost 2017;43(06):562-571

9 Selvam S, James P. Angiodysplasia in von Willebrand disease: understanding the clinical and basic science. Semin Thromb Hemost 2017;43(06):572-580

10 Muczynski V, Christophe OD, Denis CV, Lenting PJ. Emerging therapeutic strategies in the treatment of hemophilia A. Semin Thromb Hemost 2017;43(06):581-590

11 Stavropoulos K, Imprialos KP, Bouloukou S, Boutari C, Doumas M. Hematocrit and stroke: a forgotten and neglected link? Semin Thromb Hemost 2017;43(06):591-598

12 Arachchillage DRJ, Laffan M. Can mean platelet volume (MPV) be used as a predictor of vascular disease? Problems and pitfalls. Semin Thromb Hemost 2017;43(06):599-608

13 Lippi G, Danese E, Mattiuzzi C, Favaloro EJ. The intriguing link between the intestinal microbiota and cardiovascular disease. Semin Thromb Hemost 2017;43(06):609-613

14 Dentali F, Gessi V, Marcucci R, Gianni M, Grandi AM, Franchini M. Lipoprotein(a) as a risk factor for venous thromboembolism: a systematic review and meta-analysis of the literature. Semin Thromb Hemost 2017;43(06):614-620

15 Lippi G, Salvagno GL, Danese E, et al. The serum concentration of growth differentiation factor- 15 is independently associated with global platelet function and higher fibrinogen values in adult healthy subjects. Semin Thromb Hemost 2017;43(06): 621-628

16 Liu Q, Xu H, Guan X, et al. Clinical significance of antinuclear and anti-extractable nuclear antigen antibody in childhood immune thrombocytopenia. Semin Thromb Hemost 2017;43(06):629-634 